\title{
Reply to 'Cardiac remodeling after reduction of high-flow arteriovenous fistulas in end-stage renal disease: methodological issues'
}

Hypertension Research (2017) 40, 411; doi:10.1038/hr.2016.160; published online 1 December 2016

We thank Dr Ayubi and colleagues for their interest and comments regarding our recent study ${ }^{2}$ demonstrating in patients with high-flow arteriovenous fistulas that the effect of arteriovenous fistula (AVF) flow reduction on heart remodeling is dependent on cardiac index before operation, but does not depend on increased AVF flow.

Ayubi et al. ${ }^{1}$ have raised concerns regarding the statement that 'the left ventricular enddiastolic diameter decrease after operation is predicted by baseline left ventricular diastolic diameter', which is mentioned in the discussion section. This statement is not a result of the present study, but that of a study among patients after kidney transplantation, ${ }^{3}$ in which the magnitude of left ventricular (LV) mass reduction was independent of baseline AVF flow, but correlated with baseline LV mass and diameter. This study is discussed, because it supports our result that AVF flow is not able to predict LV changes after AVF reduction.

We agree with the authors regarding the comment that dichotomization of a continuous variable may have an effect on the results, because it decreases statistical power, ${ }^{4}$ but it does not lead to regression to the mean. Furthermore, in clinical practice a dichotomized variable is needed to draw conclusion about the necessity to intervene.
In the present study, cardiac index cutoff value was based on accepted values. Constructing an receiver operating characteristic curve, as suggested by the authors of the comment, would require dichotomization of the left ventricular end-diastolic diameter change, which would bring another source of bias. While cardiac index is a subject of intra-individual variability, so is the AVF flow.

In the present study, a mixed linear model with cardiac index group, time and their interaction as fixed variables was used; thus, these models were not overparameterized to the sample size.

\section{CONFLICT OF INTEREST}

Drs PB and SR are owners of the BalRok clamp registration in the Office for Harmonization in the Internal Market (OHIM) Trade Marks and Designs e Community Design, RCD application number: 002024539. The remaining authors declare no conflict of interest.

Peter Wohlfahrt ${ }^{1,2,3}$, Slavomir Rokosny ${ }^{4}$, Vojtech Melenovsky ${ }^{2}$, Barry A Borlaug ${ }^{5}$, Vera Pecenkova ${ }^{2}$ and Peter Balaz ${ }^{4,6,7}$

${ }^{1}$ Center for Cardiovascular Prevention of the First Faculty of Medicine, Charles University and Thomayer Hospital, Prague, Czech Republic; ${ }^{2}$ Department of Cardiology,
Institute for Clinical and Experimental Medicine-IKEM, Prague, Czech Republic;

${ }^{3}$ International Clinical Research Center, St Anne's University Hospital, Brno, Czech Republic; ${ }^{4}$ Vascular and Transplant Surgery Department, Institute for Clinical and Experimental Medicine-IKEM, Prague, Czech Republic; ${ }^{5}$ Division of Cardiovascular Diseases, Department of Medicine, Mayo Clinic Rochester, Rochester, MN, USA; ${ }^{6}$ Department of Transplantation Renal Unit, Guy's Hospital, London, UK and

${ }^{7}$ Department of Surgery, Faculty Hospital Kralovske Vinohrady, 3rd Medical Faculty, Charles University, Prague, Czech Republic E-mail:wohlfp@gmail.com

1 Ayubi E, Khazaei S, Doosti-Irani A, Safiri S. Cardiac remodeling after reduction of high-flow arteriovenous fistulas in end-stage renal disease: methodological issues. Hypertens Res 2017; 40: 412.

2 Wohlfahrt P, Rokosny S, Melenovsky V, Borlaug BA, Pecenkova V, Balaz P. Cardiac remodeling after reduction of high-flow arteriovenous fistulas in end-stage renal disease. Hypertens Res 2016; 39: 654-659.

3 Unger $\mathrm{P}$, Xhaet $\mathrm{O}$, Wissing KM, Najem B, Dehon P, van de Borne $P$. Arteriovenous fistula closure after renal transplantation: a prospective study with 24-h ambulatory blood pressure monitoring. Transplantation 2008; 85: 482-485.

4 Altman DG, Royston P. The cost of dichotomising continuous variables. BMJ 2006; 332: 1080. 